\title{
A mulher e a dependência química: que relação é essa?
}

Women and chemical dependency: what is this relationship?

\section{Ana Paula Farias Cezar', Ingrid D'Avila Francke ${ }^{2}$ e Tiago da Rocha Ribeiro}

Resumo: $O$ consumo de substâncias psicoativas por mulheres vem aumentando expressivamente nos últimos anos, porém, não acompanha o aumento proporcional de estudos sobre o consumo para esse público ou de procura por atendimentos especializados. Em lugar disso, não somente essas mulheres procuram atendimento tardiamente e em decorrência, muitas vezes, de comorbidades físicas como também são estigmatizadas no meio que estão inseridas. Normalmente, somente depois de inseridas no serviço, revelam o seu uso/dependência química devido ao medo de serem hostilizadas também no ambiente de tratamento. Sendo que essa procura tardia pode ser associada a dificuldade do reconhecimento da dependência em razão dos estigmas a ela associados ou da falta de apoio familiar para o enfrentamento da situação. Foi desenvolvida uma revisão sistemática da literatura buscando entender quais são as principais necessidades desse público e como a dependência química se manifesta neste caso. Dessa forma, foi possível perceber a escassez de artigos que tivessem como foco o atendimento às mulheres e que incluíssem intervenções direcionadas a elas. No entanto, estudos encontrados incentivam os tratamentos humanizados e que se continue pesquisando as necessidades da mulher dependente química a fim de que a mesma receba oportunidade de participar de forma ativa em seu tratamento.

Palavras-chave: Mulheres; Dependência química; Perfil.

\begin{abstract}
The consumption of psychoactive substances by women has increased significantly in recent years, however, it does not follow the proportional increase of studies on the consumption of this public or the demand for specialized care. Instead, not only do these women seek care late and often as a result of physical comorbidities, but they are also stigmatized in their environment. Usually, only after being inserted in the service, they reveal their chemical use/dependence due to the fear of being harassed in the treatment environment as well. This late search may be associated with the difficulty of recognizing the dependency due to the stigmas associated with it or the lack of family support to face the situation. A systematic review of the literature was developed in an attempt to understand what are the main needs of this public and how chemical dependency manifests itself in this case. Thus, it was possible to notice the scarcity of articles that focused on the care of women and that included interventions directed to them. However, the studies found encourage humanized treatments and to continue researching the needs of chemically dependent women so that they are given the opportunity to actively participate in their treatment.
\end{abstract}

Keywords: Women; Chemical dependency; Profile.

\footnotetext{
1 Acadêmica do Curso de Psicologia. Universidade Luterana do Brasil - Campus Guaíba. E-mail: ana.cezar@rede.ulbra.br

2 Psicóloga. Doutora em Saúde Coletiva pela Unisinos. Docente da Universidade Luterana do Brasil - Campus Guaíba. E-mail: ingrid.francke@ulbra.br

3 Psicólogo. Especializando em Terapia Cognitivo-Comportamental, Universidade Luterana do Brasil - Campus Guaíba. E-mail: tiagoribeiro@rede.ulbra.br
} 


\section{Introdução}

Para Silva, Carbonera e Prado (2014) a dependência química compõe um grande desafio à sociedade como um todo, sendo esse um grande problema de saúde, segurança assistência pública. Os autores ainda lembram o fato de que a dependência química não se resume ao uso de Substâncias Psicoativas (SPA), mas que também pode acarretar em problemas como acidentes de trânsito, violências, comorbidades com transtornos mentais, etc. E, trazem por fim que, muitas vezes, essas mulheres buscam tratamento não para a dependência química e sim para essas outras questões citadas.

Mas, algumas pesquisas nos mostram que não somente as mulheres têm dificuldade em reconhecer o problema em si mesmas, como também ainda é pouco estudada a dependência química em mulheres -mesmo que o consumo entre esse público esteja aumentando- e as diferentes implicações quando pensamos na diferença entre esse grupo e o grupo masculino (Fertig et al., 2016; Pedroso, Kessler \& Pechansky, 2013 citado por Madalena \& Sartes, 2018). Ou seja, temos então destacado um dos motivos da importância de darmos atenção a este tema. Além disso, segundo Sarmiento et al. (2018) é de suma importância entendermos a que público o tratamento se destina a fim de atender suas demandas específicas e proporcionar o tratamento mais humanizado possivel.

Por outro lado, em linhas gerais, segundo Freire, Melo, Carvalho, Melo e Limeira (2016), torna-se papel do profissional da saúde conhecer e respeitar a singularidade da mulher e sua família. Ou seja, eles finalizam explicando que esse éo modo de entender que relação aquela mulher teceu com a substância e como a família encara tal situação (o profissional tenderá a trabalhar com a família também). Sendo que tais apontamentos veem de encontro ao que traz Freire et al. (2016), o qual conclui seu estudo com mulheres-mães adictas com a ideia de que é essencial a participação da família no tratamento. Isto é, os autores nos trazem que desse modo além de se criar uma aproximação entre paciente e família se tem um enfraquecimento dos estigmas e preconceitos que cercam esse contexto. Isso também pode ser relacionado ao que diz Fertig et al. (2016), o qual preconiza que se minimizem as intervenções unicamente medicamentosas para que as pacientes possam ser sujeitos ativos em seu tratamento, envolvidas com as estratégias desenvolvidas por sua equipe médica de referência.

Logo, podemos inferir que essas são algumas das justificativas para que se estude o público feminino e as possibilidades de inserção nesse meio. Isto é, essa pesquisa poderá auxiliar em futuras pesquisas que visem o desenvolvimento de políticas públicas para que mais mulheres possam se beneficiar de tratamentos em serviços especializados. Logo, se tem como objetivo geral conhecer o perfil das pacientes adictas que frequentam centros de tratamentos especializados. E como objetivos específicos se tem: identificar o perfil das adictas conforme a droga de escolha; verificar as dificuldades enfrentadas por estas mulheres durante o tratamento; conhecer os gatilhos para recaída; comparar os perfis das adictas com as linhas de cuidados oferecidas a elas.

\section{Método}

Trata-se de uma pesquisa de revisão sistemática de caráter descritivo das informações. Por não se ter a ideia de fazer metanálise e sim descrever as informações relevantes aos objetivos deste estudo, será de abordagem qualitativa. Tal metodologia se enquadra na descrita por Stevens (2001 citado por Santos, Pimenta \& Nobre, 2007), a qual traz a ideia de que estudos assim estruturados objetivam sintetizar dados de estudos primários. Ou seja, estaremos abordando estudos que se enquadram na prática básica em evidências de uma maneira padronizada e que então (podemos inferir) poderá ser reproduzida por outras pessoas que sigam o mesmo processo, diferente da revisão narrativa, a qual os autores alertam para possivivel tendenciosidade.

Assim sendo, temos como alvo deste estudo: artigos cientíícos focados no público feminino e em tratamento para dependência química. Logo, se tem como critérios de inclusão: ter sido publicado nos últimos dez anos e ser revisado por pares. Porém, como critérios de exclusão se têm: serem artigos de revisão; afastar-se dos objetivos desse estudo; ter como público-alvo apenas homens ou abordar mulheres em dependência química, mas que não estão/ estiveram em tratamento da dependência; artigos duplicados. Ao mesmo tempo, quanto à coleta dos dados se têm que foram consultadas as bases de dados CAPES e BVS usando as palavras-chaves: dependência química and mulheres; transtorno aditivo and mulheres; dependência química and recaída; dependência química and gatilhos; mulheres and droga de escolha. Assim, os resultados gerados podem ser observados no fluxograma abaixo:

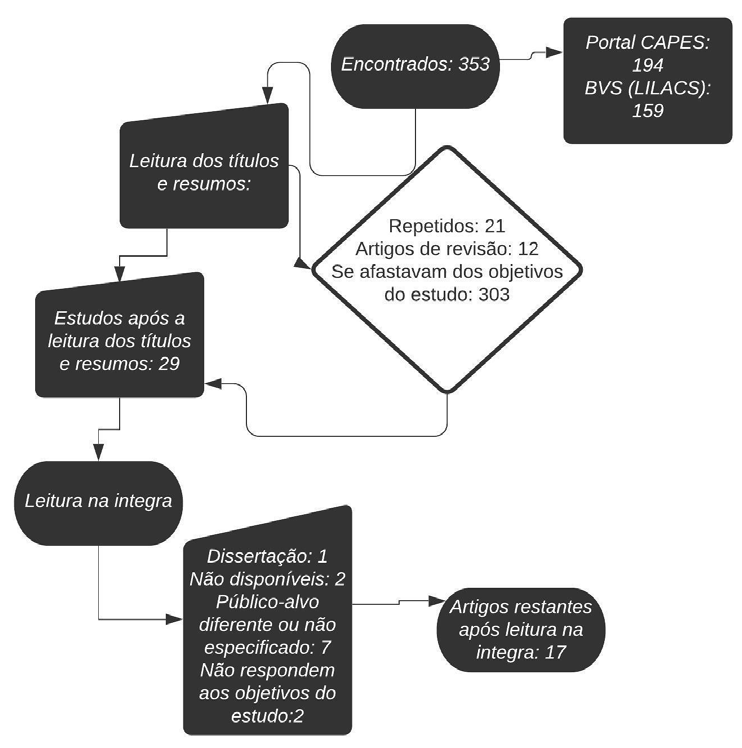

Figura 1: fluxograma criado pelas autoras do estudo.

\section{Procedimentos de análise de dados}

Os artigos elegíveis para o estudo de revisão serão tabulados em uma planilha utilizando-se o sistema de análise qual litativa segundo as perguntas $e$ objetivos de pesquisa (a partir do sistema PICO). Além disso, a busca se realizou utilizando-se os critérios de inclusão presentes no artigo e os resultados obtidos no periódico CAPES incluíram em seu filtro o critério de inclusão "revisado por pares". Porém, os resultados no periódico BVS (LLLACS) não incluíram esse critério devido ao periódico não possuir essa opção. 


\begin{tabular}{|c|c|c|c|c|c|c|}
\hline No & Título & Autores/Ano & Objetivos & $\begin{array}{l}\text { Tipo de } \\
\text { pesquisa }\end{array}$ & $\begin{array}{c}\text { Área do } \\
\text { periódico }\end{array}$ & Principais resultados \\
\hline 1 & $\begin{array}{l}\text { A Mulher no contexto } \\
\text { das Drogas: Representa- } \\
\text { çôes Sociais de Usuárias } \\
\text { em Tratamento }\end{array}$ & $\begin{array}{l}\text { Medeiros, K.T., Ma- } \\
\text { ciel, S.C. \& Sousa, } \\
\text { P.F. de. (2017) }\end{array}$ & $\begin{array}{l}\text { Analisar a dimensão representacional } \\
\text { acerca da mulher usuária de drogas } \\
\text { a partir da Teoria das Representaçôes } \\
\text { Sociais. }\end{array}$ & $\begin{array}{l}\text { Qualitativo, de ca- } \\
\text { ráter descritivo e de } \\
\text { campo. }\end{array}$ & Psicologia & $\begin{array}{l}\text { Os resultados do estudo apontam que a mulher } \\
\text { usuária de drogas é vista como uma ameaça à } \\
\text { sociedade uma vez que foge do papel conven- } \\
\text { cional imposto à mulher. Essa mulher passa a } \\
\text { ser vista como um problema e perde seu valor } \\
\text { como mulher. }\end{array}$ \\
\hline 2 & $\begin{array}{l}\text { Novas problemáticas so- } \\
\text { ciais: } 0 \text { uso do crack em } \\
\text { mulheres ea perspectiva } \\
\text { de gênero }\end{array}$ & $\begin{array}{l}\text { Limberger, J. \& An- } \\
\text { dretta, I. (2015) }\end{array}$ & $\begin{array}{l}\text { Compreender o uso do crack em mu- } \\
\text { Iheres, sua trajetória de vida e caracte- } \\
\text { rísticas clínicas, através da perspectiva } \\
\text { de gênero. }\end{array}$ & $\begin{array}{l}\text { Estudo de caso com } \\
\text { caráter qualitativo. }\end{array}$ & Psicologia & $\begin{array}{l}\text { A pesquisa aponta alguns dos problemas sociais } \\
\text { mascarados pelo uso do crack, tais como negação } \\
\text { de direitos como: falta de acompanhamento na } \\
\text { escola, falta de apoio quanto às agressões sofridas } \\
\text { e carência de informações sobre tratamento. }\end{array}$ \\
\hline 3 & $\begin{array}{l}\text { Perfil de mulheres aco- } \\
\text { Ihidas em leitos de um } \\
\text { Centro de Atenção Psi- } \\
\text { cossocial Álcool e Drogas }\end{array}$ & $\begin{array}{l}\text { Leão, N. M.f., Boska, } \\
\text { G.A., Silva, J.C. M.C., } \\
\text { Claro, H. G., Oliveira, } \\
\text { M. A. F. , \& Oliveira, } \\
\text { M. S. R. (2020) }\end{array}$ & $\begin{array}{l}\text { Caracterizar o perfil de mulheres ad- } \\
\text { mitidas para uso dos leitos de acolhi- } \\
\text { mento noturno em um CAPSad e suas } \\
\text { admissões. }\end{array}$ & $\begin{array}{l}\text { Pesquisa quantitati- } \\
\text { va de delineamento } \\
\text { retrospectivo e des- } \\
\text { critivo. }\end{array}$ & Enfermagem & $\begin{array}{l}\text { O estudo aponta que os leitos se tornam referência } \\
\text { de cuidado e proteção a essas mulheres frente } \\
\text { às suas vulnerabilidades associadas ao uso de } \\
\text { substâncias. Ademais, mesmo as pacientes } \\
\text { que recebem alta procuram o atendimento em } \\
\text { momentos de crise, } 0 \text { que indica bom vínculo } \\
\text { entre equipe e paciente. }\end{array}$ \\
\hline 5 & $\begin{array}{l}\text { Perfil dos usuários de } \\
\text { crackna mídia brasileira: } \\
\text { análise de um jornal e } \\
\text { duas revistas de edição } \\
\text { nacional. }\end{array}$ & $\begin{array}{l}\text { Zanotto, D. F., \& As- } \\
\text { sis, F. B. (2017) }\end{array}$ & $\begin{array}{l}\text { Analisar o perfil dos usuários de crack } \\
\text { por meio de reportagens de um jornal e } \\
\text { de duas revistas de circulação nacional. }\end{array}$ & $\begin{array}{l}\text { Pesquisa qualitativa } \\
\text { e delineamento do- } \\
\text { cumental. }\end{array}$ & Saúde coletiva & $\begin{array}{l}\text { A mídia que foi apresentada nesse estudo se faz } \\
\text { presente como formadora de opinião pública e } \\
\text { legitimiza os discursos da população referente } \\
\text { a uma negativa do perfil dos usuários de crack } \\
\text { e de que é necessária uma política higienizadora } \\
\text { dos bairros. }\end{array}$ \\
\hline 6 & $\begin{array}{l}\text { Assertividade em mu- } \\
\text { Iheres dependentes de } \\
\text { crack }\end{array}$ & $\begin{array}{l}\text { Souza, M. C. H., } \\
\text { Mühlen, B. K. V., Co- } \\
\text { elho, L. R. M., Olivei- } \\
\text { ra, C. P. , Rodrigues, } \\
\text { V. S., Oliveira, M.S. } \\
\text { \& Strey, M. N. (2014) }\end{array}$ & $\begin{array}{l}\text { Averiguar a assertividade de mulheres } \\
\text { dependentes de crack, durante a sessão } \\
\text { "Recusas Eficazes", em uma comunida- } \\
\text { de terapêutica. }\end{array}$ & $\begin{array}{l}\text { Pesquisa qualitativa } \\
\text { com delineamento } \\
\text { exploratório. }\end{array}$ & Psicologia & $\begin{array}{l}\text { Através desse estudo foi possível compreender } \\
\text { como as mulheres com dependência em crack } \\
\text { vivenciam o seu uso e como percebem suas } \\
\text { habilidades de enfrentamento e assertividade } \\
\text { ante ao estímulo da venda de drogas. }\end{array}$ \\
\hline 7 & $\begin{array}{l}\text { Análise de determinan- } \\
\text { tes intra e interpessoais } \\
\text { como motivos de recaída } \\
\text { no contexto da depen- } \\
\text { dência química }\end{array}$ & $\begin{array}{l}\text { Buriola, A. A., Silva, } \\
\text { A. S. T., Prestes, A. H. } \\
\text { 0., Nascimento, L. A. } \\
\text { S., Cavelleri, M. Z., \& } \\
\text { Bordão, F.C.C(2018) }\end{array}$ & $\begin{array}{l}\text { Identificar os motivos de recaída de } \\
\text { dependentes químicos, em tratamento } \\
\text { em um Centro de Atenção Psicossocial } \\
\text { para álcool e drogas. }\end{array}$ & $\begin{array}{l}\text { Pesquisa qualitativa } \\
\text { de abordagem des- } \\
\text { critiva e exploratória. }\end{array}$ & Enfermagem & $\begin{array}{l}\text { Os autores têm como expectativa que esse estudo } \\
\text { auxilie no processo de reabilitação dos pacientes } \\
\text { uma vez que o mesmo versa sobre a recaída e este } \\
\text { é um tema pertinente ao tratamento. }\end{array}$ \\
\hline 8 & $\begin{array}{l}\text { A Entrevista Motivacio- } \\
\text { nal em Adolescentes } \\
\text { Usuários de Droga que } \\
\text { Cometeram Ato Infra- } \\
\text { cional }\end{array}$ & $\begin{array}{l}\text { Andreatta, I. \& } \\
\text { Oliveira, M. da S. } \\
\text { (2011) }\end{array}$ & $\begin{array}{l}\text { Verificar a efetividade da Entrevista } \\
\text { Motivacional (EM) em adolescentes } \\
\text { que cometeram ato infracional, usuários } \\
\text { de drogas. }\end{array}$ & $\begin{array}{l}\text { Estudo clínico, com } \\
\text { delineamento expe- } \\
\text { rimental. }\end{array}$ & Psicologia & $\begin{array}{l}0 \text { estudo demonstrou que o uso da EM e da psi- } \\
\text { coeducação foram efetivos quanto a diminuiç̧ão } \\
\text { do consumo de drogas (verificado na avaliação } \\
\text { e reavaliação dos pacientes) e de avanço nos } \\
\text { estágios de prontidão para mudança (observada } \\
\text { pela escala URICA). }\end{array}$ \\
\hline 9 & $\begin{array}{l}\text { Crack e sonhos: a visão } \\
\text { dos usuários }\end{array}$ & $\begin{array}{l}\text { Silva, T. R. \& Nappo, } \\
\text { S. A. (2019) }\end{array}$ & $\begin{array}{l}\text { Descrever os conteúdos dos sonhos } \\
\text { dos usuários de crack, de acordo com } \\
\text { sua visão, e analisar a interferência que } \\
\text { produzem no processo de abstinência. }\end{array}$ & Qualitativa. & Saúde coletiva & $\begin{array}{l}0 \text { estudo aponta que, em geral, os sonhos dos } \\
\text { usuários os fazem reviver todo o processo do uso } \\
\text { do crack, incluindo cheiros e lugares. Porém, os } \\
\text { autores também apontam como sendo de maior } \\
\text { relevância o significado atribuído pelos pacientes } \\
\text { a cada um de seus sonhos. }\end{array}$ \\
\hline 10 & $\begin{array}{l}\text { A carreira moral da } \\
\text { vergonha na visão de } \\
\text { homens e mulheres "al- } \\
\text { coólatras" }\end{array}$ & Alzuguir, F.V. (2014) & $\begin{array}{l}\text { Analisar a carreira moral de tratamento } \\
\text { do alcoolismo através de depoimentos } \\
\text { de homens e mulheres, em tratamento, } \\
\text { sobre a moral da vergonha. }\end{array}$ & Qualitativa. & Saúde coletiva & $\begin{array}{l}\text { O estudo apontou relação própria entre vergonha, } \\
\text { responsabilização e conscientização do alcoolis- } \\
\text { mo. Então, o alcoolista percebendo-se doente } \\
\text { tendea sentir vergonha de seus comportamentos, } \\
\text { buscar internaçãa e uso de medicaçãa, porém, } \\
\text { não mais em uma posição de estigmatização e } \\
\text { culpabilização. }\end{array}$ \\
\hline 11 & $\begin{array}{l}\text { Das proposições da } \\
\text { política às práticas dos } \\
\text { serviços: há novidades } \\
\text { nos Centros de Atenção } \\
\text { Psicossocial Álcool e } \\
\text { Drogas? }\end{array}$ & $\begin{array}{l}\text { Machado, A.. R., Mo- } \\
\text { dena, C. M., \& Luz, Z. } \\
\text { M. P. (2020) }\end{array}$ & $\begin{array}{l}\text { Compreender como têm sido desen- } \\
\text { volvidas as práticas desses serviços, } \\
\text { CAPS-ad, bem como a lógica de atenção } \\
\text { que adotam. }\end{array}$ & Qualitativa. & Saúde coletiva & $\begin{array}{l}\text { A pesquisa mostra que o CAPS-ad tem cumprido } \\
0 \text { que se propõe quanto ao uso de drogas, ou } \\
\text { seja, tem aberto oportunidades às pessoas que } \\
\text { usam drogas. }\end{array}$ \\
\hline
\end{tabular}




\begin{tabular}{|c|c|c|c|c|c|c|}
\hline No & Título & Autores/Ano & Objetivos & $\begin{array}{l}\text { Tipo de } \\
\text { pesquisa }\end{array}$ & $\begin{array}{c}\text { Área do } \\
\text { periódico }\end{array}$ & Principais resultados \\
\hline 12 & $\begin{array}{l}\text { Uso de substâncias psi- } \\
\text { coativas em mulheres } \\
\text { em tratamento ambu- } \\
\text { latorial }\end{array}$ & $\begin{array}{l}\text { Santos, J. A. T., Per- } \\
\text { ruchi, L.G., Pegoraro } \\
\text { N. P. J., Scherer, Z. A. } \\
\text { P., Souza, J., Santos, } \\
\text { M. A. \& Pillon, S. } \\
\text { C.(2019) }\end{array}$ & $\begin{array}{l}\text { Caracterizar o perfil sociodemográfico e } \\
\text { psiquiátrico de usuárias de substâncias } \\
\text { psicoativas em tratamento para depen- } \\
\text { dência química. }\end{array}$ & $\begin{array}{l}\text { Estudo descritivo de } \\
\text { abordagem quan- } \\
\text { titativa. }\end{array}$ & Enfermagem & $\begin{array}{l}0 \text { estudo mostra que essas mulheres têm alto } \\
\text { nível de morbidade e acentuada vulnerabilidade } \\
\text { psicossocial, os quais juntamente com os sintomas } \\
\text { associados ao uso de substâncias devem receber } \\
\text { minuciosa investigação. }\end{array}$ \\
\hline 13 & $\begin{array}{l}\text { Motivos da recaída do } \\
\text { uso de drogas por mu- } \\
\text { Iheres na perspectiva da } \\
\text { Fenomenologia Social }\end{array}$ & $\begin{array}{l}\text { Soccol, K. L.S., Terra, } \\
\text { M. G., Ribeiro, D. B., } \\
\text { Siqueira, D. F., Lac- } \\
\text { chini, A. J. B. \& Ca- } \\
\text { nabarro, J. L.(2019) }\end{array}$ & $\begin{array}{l}\text { Compreender a intencionalidade da } \\
\text { ação à recaída ao uso de drogas por } \\
\text { mulheres assistidas em um Centro de } \\
\text { Atenção Psicossocial Álcool e Drogas. }\end{array}$ & $\begin{array}{l}\text { Qualitativa de abor- } \\
\text { dagem Fenomeno- } \\
\text { logia Social. }\end{array}$ & Enfermagem & $\begin{array}{l}0 \text { estudo apontou a necessidade de os profissio- } \\
\text { nais da saúde trabalharem questões de resiliência } \\
\text { com essas pacientes no que se refere ao enfren- } \\
\text { tamento das questões de vida diárias e que estes } \\
\text { auxiliem a despertar } 0 \text { interesse das mulheres } \\
\text { em atividades que lhes tragam prazer e alegria. }\end{array}$ \\
\hline 14 & $\begin{array}{l}\text { Caracterização de mu- } \\
\text { Iheres menores de idade } \\
\text { em um centro especia- } \\
\text { lizado em dependência } \\
\text { química }\end{array}$ & $\begin{array}{l}\text { Quirós, M. del R. M } \\
\text { (2016) }\end{array}$ & $\begin{array}{l}\text { Caracterizar as mulheres menores de } \\
\text { idade em um centro especializado em } \\
\text { dependência química durante os anos } \\
\text { de } 2009 \text { e } 2014 \text {. }\end{array}$ & $\begin{array}{l}\text { Retrospectivo des- } \\
\text { critivo com enfoque } \\
\text { quantitativo. }\end{array}$ & Enfermagem & $\begin{array}{l}0 \text { estudo apontou a necessidade de se realizarem } \\
\text { outras pesquisas capazes de reforçar a atenção ao } \\
\text { tema e diminuir a demanda e danos causados } \\
\text { pelo uso e abuso de substâncias psicoativas. }\end{array}$ \\
\hline 15 & $\begin{array}{l}\text { Barreiras e facilidades } \\
\text { para buscar tratamento } \\
\text { especializado encon- } \\
\text { tradas por mulheres } \\
\text { que usam substâncias } \\
\text { psicoativas }\end{array}$ & $\begin{array}{l}\text { Albuquerque, C.S. } \\
\text { \& Nóbrega, M. P. S. } \\
\text { S. (2016) }\end{array}$ & $\begin{array}{l}\text { Identificar as barreiras e amenidades } \\
\text { encontradas pelas mulheres ao bus- } \\
\text { carem tratamento para dependência } \\
\text { química. }\end{array}$ & Quantitativo. & Psicologia & $\begin{array}{l}0 \text { estudo apontou características que facilitam } \\
\text { ou dificultam o acesso das mulheres aos serviços } \\
\text { especializados em tratamento de dependência } \\
\text { química, } 0 \text { que evidenciou debilidades na di- } \\
\text { vulgação dos serviços e nos encaminhamentos } \\
\text { das mulheres ao tratamento diante de detecção } \\
\text { precoce da dependência. }\end{array}$ \\
\hline 16 & $\begin{array}{l}\text { Quem procura tratamen- } \\
\text { to para abuso de subs- } \\
\text { tância na rede pública no } \\
\text { Brasil? Resultados de um } \\
\text { estudo multicêntrico en- } \\
\text { volvendo quatro capitais } \\
\text { brasileiras }\end{array}$ & $\begin{array}{l}\text { Faller, S., Peuker, A. } \\
\text { C., Sordi, A., Stolf, A., } \\
\text { Souza-Formigoni, A. } \\
\text { L., Cruz, M. S. Brasi- } \\
\text { liano, S., Pechansky, } \\
\text { F. \& Kessler, F(2014) }\end{array}$ & $\begin{array}{l}\text { Avaliar as características dos usuários de } \\
\text { álcool e drogas que buscam atendimen- } \\
\text { to no Sistema Único de Saúde do Brasil. }\end{array}$ & $\begin{array}{l}\text { Quantitativo, do tipo } \\
\text { transversal multi- } \\
\text { cêntrico. }\end{array}$ & Psiquiatria & $\begin{array}{l}0 \text { estudo apontou a importância de se levarem em } \\
\text { conta durante o tratamento questões geradoras } \\
\text { de preocupações aos pacientes, tais como relações } \\
\text { familiares, emprego e educação. }\end{array}$ \\
\hline 17 & $\begin{array}{l}\text { Profile of women drug } \\
\text { addicts treated at the } \\
\text { psychosocial care center } \\
\text { alcohol and other drugs: } \\
\text { documentary study }\end{array}$ & $\begin{array}{l}\text { Lima, H.P., Macedo, } \\
\text { J. Q., Braga, V. A.B. } \\
\text { Lemos \& Júnior, I. } \\
\text { A.S(2011) }\end{array}$ & $\begin{array}{l}\text { Traçar o perfil de mulheres dependentes } \\
\text { de drogas tratados em CAPS-ad no } \\
\text { Ceará. }\end{array}$ & $\begin{array}{l}\text { Quantitativa, do tipo } \\
\text { documental e retros- } \\
\text { pectivo. }\end{array}$ & Enfermagem & $\begin{array}{l}0 \text { artigo apontou algumas das vulnerabilidades } \\
\text { das mulheres e como isso se reflete no seu trata- } \\
\text { mento. } 0 \text { que os autores nos trazem como sendo } \\
\text { indicativo da importância de se estabelecerem } \\
\text { planos terapêuticos focados nessa população } \\
\text { dando ênfase a questões como promoção da } \\
\text { saúde, autoestima e reintegração social. }\end{array}$ \\
\hline
\end{tabular}

Tabela 1 - Criada pelas autoras do estudo.

\section{Resultados}

Após analisar como os achados contribuem para esta pesquisa buscou-se agrupar as respostas em sessões referentes aos objetivos do estudo (a partir dos estudos que respondem um ou outro objetivo da presente revisão), a saber: perfil das adictas; dificuldades para acessar o serviço e/ou durante o tratamento; gatilhos para recaídas; como os serviços se propõem a tratar essas mulheres.

\section{Perfil das adictas}

A menor faixa etária apresentada foi a de Quirós (2016), com as pacientes entre 15 a 17 anos, usuárias de múltiplas drogas, mas com predomínio da maconha, tabaco álcool -empatado o uso com o da cocaína-. Depois, seguindo a análise das faixas etárias temos as idades apontadas, respectivamente, de 15 a 65 anos, sendo as drogas de escolha em sua maioria: álcool, cocaína, cracke maconha (Albuquerque \& Nóbrega, 2016); 18 a 40 anos, a droga de escolha temos como as lícitas: álcool, tabaco e psicotrópicos e drogas ilícitas temos: maconha, cracke cocaína. (Lima, Macedo, Braga, Lemos \& Júnior, 2011). Nas faixas etárias de 20 a 60 anos, sem especificação de droga (Soccol et al., 2019); de 18 a 73 anos, uso álcool, maconha, crack e cocaína (Santos et al., 2019); 25 anos, uso de crack (Limberger \& Andretta, 2015). Enas maiores médias se tem de 36 anos, uso de múltiplas drogas, incluindo crack e cocaína (Vargas, Ponce, Ramírez, Pereira \& Nóbrega, 2018; Faller et al., 2014) e 38 anos, com uso de crack e álcool (Leão et al., 2020).

Além disso, sobre o nível educacional das pacientes: ou o estudo não o citava ou dizia que as mulheres possuíam baixo nível de escolaridade, em sua maioria com ensino fundamental incompleto. Enquanto que sobre a informação socioeconômica estava sendo descrita como"sem exercer atividade remunerada/desempregada" (Lima et al., 2011; Albuquerque \& Nóbrega, 
2016; Santos et al., 2019; Vargas et al., 2018; Leão et al., 2020). No entanto, no estudo de Limberger e Andretta (2015) a paciente estava trabalhando como empregada doméstica, no estudo de Quirós (2016) a variável sociodemográfica não tinha correlação com o uso de substâncias psicoativas e nos outros estudos não estava especificada a ocupação das mulheres (Soccol et al, 2019; Faller et al., 2014).

Ademais, a alguns dos estudos apontavam que as pacientes, em sua maioria eram solteiras (Lima et al., 2011; Santos et al., 2019; Vargas et al., 2018; Leão etal, 2020; Limberger \& Andretta, 2015) e viviam em situação de vulnerabilidade quanto a moradia, muitas morando nas ruas (Vargas et al., 2018; Leão et al., 2020), com familiares, amigos ou residenciais/comunidade terapêutica (Lima et al, 2011; Souza etal., 2014; Albuquerque \& Nóbrega, 2016) e um estudo apontou que a maioria possuía residência própria (Santos et al., 2019).

\section{Dificuldades para acessar o serviço e/ou durante o tratamento}

Quanto a resposta ao objetivo 2 temos que as mulheres têm relutância de entender que estão em dependência química e que precisam de tratamento especializado (Alzuguir, 2014; Soccol et al., 2019). Além disso, elas também encontram como barreiras a forma como a família as estigmatiza e não as apoia ou agem com negação em seu tratamento (Limberger \& Andretta, 2015; Quirós, 2016; Lima et al., 2011). Por isso, essas mulheres tendem a vir ao serviço com ideias pré-concebidas de que elas serão associadas a imagem marginalizada da usuária de substâncias psicoativas e tratadas como tal (Medeiros, Maciel, \& Sousa, 2017; Limberger \& Andretta, 2015; Zanotto \& Assis, 2017).

Todavia, esses receios são em parte confirmados quando essas pacientes são atendidas por profissionais que se recusam a atendê-las e/ou têm atitudes negativas em relação a elas (Albuquerque \& Nóbrega, 2016; Machado, Modena, \& Luz, 2020; Limberger \& Andretta, 2015). Porém, em meio a isso têm também a dificuldade financeira de chegar aos locais uma vez que, eventualmente, os tratamentos não são no mesmo município ou por se tratarem de menores de idade e o CAPs não suportar suas demandas do uso ou de suas comorbidades físicas (Medeiros et al., 2017; Leão et al., 2020; Vargas et al., 2018; Lima et al, 2011).

\section{Gatilhos para recaída}

As usuárias dos serviços relatam que ou fizeram seu primeiro uso em presença de alguém de sua confiança ou no uso atual o fazem junto com os companheiros amorosos e familiares (Limberger \& Andretta, 2015; Vargas et al., 2018; Albuquerque \& Nóbrega, 2016). Portanto, elas não só tendem a possuir facilidade de acesso a substância como também se reinserem no meio onde ficam vulneráveis à recaída e/ou riscos potenciais (Leão et al., 2020; Zanotto \& Assis, 2017; Souza et al., 2014; Silva \& Nappo, 2019; Soccol et al., 2019). Sendo esses gatilhos agravados caso no ambiente que a pessoa viva seja considerado comum o uso da substância. Isto é, conforme apontado por Alzuguir (2014) a vergonha seria o sentimento que sinaliza ao paciente que ele (a) está desviando do comportamento socialmente aceitável e que o protegeria contra a recaída, ao qual uma vez perdido esse sentimento, a recaída tenderia a ocorrer.
Além disso, durante o tratamento muitas delas têm sintomas de síndrome de abstinência, tais como: fissura, insônia e tremores, assim como abandonaram o tratamento precocemente ou viam na ansiedade presente na fissura um gatilho para recaída (Leão et al, 2020; Buriola et al, 2019; Silva \& Nappo, 2019). 0 que é confirmado por Soccol et al. (2019), que nos trazem que muitas pacientes recaem quando se defrontam com situações para comemorar sua alegria ou para esquecer seus problemas.

\section{Como os serviços se propõem a tratar essas mulheres?}

Zanotto e Assis (2017), ao avaliarem a ideia da população geral frente aos usuários de crack, nos trazem que a maioria das pessoas vê como sendo necessária uma política higienizadora retirando esses usuários de crack das ruas e os internando. Ou seja, os autores nos trazem que a mídia fomenta a ideia da população de que se deve usar repressão policial para se realizarem internações compulsórias baseadas no modelo biomédico de tratamento. Porém, como apontado por Vargas et al. (2018), as pacientes que procuraram tratamento em um centro especializado em SP buscaram por acolhimento e proteção. Fato esse apontado também por Albuquerque e Nóbrega (2016), os quais nos trazem que os profissionais que atendem essas pessoas não podem ter uma postura julgadora/moral em relação aos pacientes ou então acabarão reforçando os estigmas vividos fora do ambiente de tratamento.

Sendo que, além disso, Buriola et al (2019), apontam a necessidade de tratamentos ambulatoriais que se utilizem de atenção às necessidades de caráter somático e psicossocial, deixando o paciente próximo de sua realidade. Ademais, apenas o estudo de Lima et al (2011) aponta que 90\% dos prontuários de sua amostra, estava sendo atendida em razão de um plano terapêutico individual. No entanto, é imprescindível destacarmos as ações já implementadas e que foram analisadas nos estudos, em sua maioria.

Logo, em relação ao início do tratamento, Machado et al (2020) constatam que o CAPs oferece tratamento inicial aos déficits que comprometem sua saúde física e então seguem se utilizando de estratégias de redução de danos quanto ao uso das substâncias e também trabalham com reinserção social e defesa dos direitos à cidadania. Ademais, Leão et al. (2020) trazem que as pessoas em interação em leitos noturnos estão ali devido a ideia de se desintoxicarem e para minimizarem suas vulnerabilidades sociais, tal como 0 fato de morarem na rua e estarem expostos a perigos. Já em relação ao tratamento em Comunidade Terapêutica, Souza et al. (2014) apontam como sendo foco da intervenção utilizada, o role-playing, a prevenção à recaída.

Neste caso, para entrar no processo de finalizar, cabe citar Andreatta e Oliveira (2011) que nos trazem que uma estratégia que diminuiu o uso de substâncias psicoativas por adolescentes (durante atendimento em uma clínica escola) foi o uso da Entrevista Motivacional (EM). Isto é, esse decréscimo do uso foi observado após o uso de intervenções breves utilizando-se de psicoeducação sobre drogas, estímulo à motivação para mudança e uso de feedbacks da avaliação. No entanto, em consonância a todas as vantagens e desvantagens dos diferentes tipos de tratamento, algumas pesquisas apontam que os pacientes alvo do estudo combinavam distintas modalidades de atendimento, tal como atendimento em (APs e frequentar AA e NA (Alzuguir, 2014; Albuquerque \& Nóbrega, 2016; Limberger \& Andretta, 2015). 


\section{Discussões finais}

A análise dos artigos selecionados para este estudo mostrou que ainda se tem pouco material produzido, especialmente pela psicologia, que se foca exclusivamente em amostras com mulheres em tratamento para dependência química. Isto é, dos 17 artigos restantes nesta revisão sistemática apenas 4 foram advindos da psicologia com este público-alvo específico. Porém, todos os estudos selecionados abordam, de alguma forma, como a dependência química ocorre nas mulheres.

Além disso, é importante ressaltar que a forma que essas mulheres são vistas pela sociedade que estão inseridas é, basicamente, estigmatizada. Isto é, as mesmas são descritas como al guém que foge dos padrões femininos, uma má esposa e mãe. Desse modo, podemos inferir, que de tanto serem insultadas e agredidas as mesmas acabam por acreditar que realmente são do jeito que as descrevem e não procuram atendimento para a dependência química. Por isso, quando o fazem, já estão com sua saúde severamente comprometida e, consequentemente, durante o tratamento terão de lidar com a dependência química e seus agravos (comprometimentos cognitivos, por exemplo).

No entanto, todos os estudos apontaram a necessidade de tratamentos individualizados a essas pacientes. Ou seja, não somente devemos nos atentar às necessidades das pacientes em termos de sexo biológico e gênero, como também as necessidades específicas a partir da história pregressa delas. Assim sendo, embora alguns estudos criticassem o serviço onde as mulheres estavam inseridas, os autores apontavam alternativas para como lidar com aquilo. Logo, algumas conclusões que se pôde chegar a partir desta revisão incluem a necessidade de desenvolvimento de estudos que versem sobre como é feito o tratamento dessas mulheres pelo serviço de psicologia, uma vez que os mesmos ainda são escassos e as mulheres apontavam necessidades emocionais. Bem como se nota a necessidade de um trabalho de psicoeducação com os profissionais da saúde em geral buscando minimizar a estigmatização dentro dos locais de tratamento.

\section{Referências}

Albuquerque, C.S.\& Nóbrega, M. P. S. S. (2016). Barreiras e facilidades encontradas por mulheres usuárias de substâncias psicoativas na busca por tratamento especializado. Revista eletrônica saúde mental álcool e drogas, 12(1), 22-29. D0l: https://dx.doi.org/10.11606/issn.1806-6976. v12i1p22-29

Alzuguir, F. V. (2014). A carreira moral da vergonha na visão de homens e mulheres "alcoólatras". Physis, 24 (01) D0I: https://doi.org/10.1590/ S0103-73312014000100002

Andreatta, I. \& Oliveira, M. S. (2011). Entrevista motivacional em adolescentes usuários de drogas que cometeram ato infracional. Psicologia: Reflexão e Crítica, 24(2), 218-226. D0l: http://doi.org/10.1590/S010279722011000200002

Buriola, A. A., Silva, A. S. T., Prestes, A. H. O., Nascimento, L. A. S., Cavelleri, M. Z., \& Bordão, F.C.C. (2018). Análise de determinantes intra e interpessoais como motivos de recaída no contexto da dependência química. Journal of Nursing and Health. 8(2), 1-15. D0l: http://doi.Org/10.15210/Jonah. V8i2.14022

Faller, S., Peuker, A. C., Sordi, A., Stolf, A., Souza-Formigoni, A. L., Cruz, M. S. Brasiliano, S., Pechansky, F. \& Kessler, F. (2014). Quem procura tratamento para abuso de substância na rede pública no Brasil? Resultados de um estudo multicêntrico envolvendo quatro capitais brasileiras. Trends Psychiatry Psychother, 36 (4) DOl: https://doi.org/10.1590/2237-6089-2014-0040
Fertig, A.; Schneider, J. F.; Oliveira, G.C. De; Olschowsky, A.; Camatta, M. W.; Pinho, L. B. (2016). Mulheres usuárias de crack: Conhecendo suas histórias de vida. Esc. Anna Nery, 20 (2). Recuperado de: https://www.scielo.br/j/ ean/a/LTrHJcVyY8rRGKJ8VB6Hz5w/?lang=pt\#.

Freire, A. K. Da S.; Melo, M. C. P.; Carvalho, M. F. A. A; Melo, R. S. De; Limeira, \& C. G. (2016). Configurações e dinâmicas familiares de mulheres-mães durante trajetória na dependência química. Ciencia y Enfermería, 22 (2): 51-62. Recuperado de: https://scielo.conicyt.cl/pdf/cienf/v22n2/ art_05.pdf

Leão, N. M.f., Boska, G.A., Silva, J. C. M. C., Claro, H. G., Oliveira, M. A. F.;\& Oliveira, M. S. R. (2020). Perfil de mulheres acolhidas em leitos de um Centro de Atenção Psicossocial Álcool e Drogas. Enfermagem em foco, 11(1), 63-68. D0l: https://doi.org/10.21675/2357-707X.2020.v11.n1.2528

Lima, H.P., Macedo, J. Q., Braga, V. A.B. Lemos; \& Júnior, I. A.S. (2011). Profile of women drug addicts treated at the psychosocial care center alcohol and other drugs: documentary study. Online Brazilian Journal of Nursing, 10(2), 2-15. DOl: https://doi.org/10.5935/1676-4285.20113257

Limberger, J.; \& Andretta, I. (2015). Novas problemáticas sociais: 0 uso do crack em mulheres e a perspectiva de gênero. Revista (S, 15, 42-65. D01: https://doi.org/10.18046/recs.115.1965

Machado, A. R., Modena, C. M.; \& Luz, Z. M. P. (2020). Das proposições da política às práticas dos serviços: há novidades nos (entros de Atenção Psicossocial Álcool e Drogas?. Physis, 30(01). D0l: https://doi.org/10.1590/ S0103-73312020300118

Madalena,T. S. \& Sartes, L. M. A. (2018). Usuários de crack em tratamento em Comunidades Terapêuticas: perfil e prevalência.Arquivos Brasileiros de Psicologia, 70(1), 21-36. Recuperado de http://pepsic.bvsalud.org/scielo.php?script=sci_arttext\&pid=\$1809-52672018000100003\&lng=pt\&nrm=iso

Medeiros, K. T., Maciel, S. C. \& Sousa, P., F. (2017). A Mulher no Contexto das Drogas: Representações Sociais de Usuárias em Tratamento. Paidéia, 27(1), 439-447. DOl: https://doi.org/10.1590/1982-432727s1201709

Quirós, M. R. M (2016). Caracterização de mulheres menores de idade em um centro especializado em dependência química. Journal Health NPEPS, 1(1). 4-16. Recuperado de https://periodicos.unemat.br/index.php/jhnpeps/ article/view/1563

Santos, J. A. T., Perruchi, L.G., Pegoraro N. P. J., Scherer, Z. A. P., Souza, J., Santos, M. A. \& Pillon, S. C. (2019). Uso de substâncias psicoativas em mulheres em tratamento ambulatorial. Rev. Bras. Enfermagem, 72 (3) D0l: https:// doi.org/10.1590/0034-7167-2018-0399

Santos, C. M. Da C; Pimenta, C. A. De M.; Nobre, M. R. C. (2007). A estratégia PICO para a construção da pergunta de pesquisa e busca de evidências. Rev Latino-am Enfermagem, 15(3). 1-4. Recuperado em: https://www. scielo.br/pdf/rlae/v15n3/pt_v15n3a23.pdf.

Sarmiento, Y. E. S.; Gonçalves, N. N.; Vaz, C.; Neiva, G. D.; Rodrigues, G. C.; Oliveira, J.S. \& Aguiar, C.A. (2018). Dependência química e gênero: um olhar sobre as mulheres. Núcleo de estudos de gênero: caderno espaço feminino, 31(2), 149-160. D0l: http://dx.doi.org/10.14393/CEF-v31n2-2018-8

Silva, T. R. \& Nappo, S. A. (2019). Crack e sonhos: a visão dos usuários. Ciência e Saúde Coletiva, 24 (3). D0l: https://doi.org/10.1590/141381232018243.05072017

Silva, A. F., Carbonera, A. \& Prado, J. A. F. A. (2014). A luta de uma mulher contra a dependência química: Um olhar da Gestalt-terapia. Revista IGT na Rede, 11(21). 297-307. Recuperado de: http://pepsic.bvsalud.org/pdf/ igt/v11n21/v11n21a05.pdf

Soccol, K. L. S., Terra, M. G., Ribeiro, D. B., Siqueira, D. F., Lacchini, A. J. B. \& Canabarro, J. L. (2019). Motivos da recaída ao uso de drogas por muIheres na perspectiva da Fenomenologia Social. Enfermagem em Foco, 10(15), 118-122. D0l: https://doi.org/10.21675/2357-707X.2019. v10.n5.2540 
Souza, M. C. H.; Mühlen, B. K. V.; Coelho, L. R. M.; Oliveira, C. P.; Rodrigues, V. S.; Oliveira, M.S. \& Strey, M. N. (2014). Assertividade em mulheres dependentes de crack. Aletheia, (43-44), 105-115. Recuperado em http://pepsic.bvsalud.org/scielo.php?script=sci_arttext\&pi$d=\$ 1413-03942014000100008 \&$ Ing $=p t \& t$ lng $=p t$.

Vargas, D.; Ponce, T. D.; Ramírez, E. G. L.;Pereira, C. F. \& Nóbrega, M.P.S. (2018). Mulheres em tratamento especializado para uso de substâncias psicoativas: estudo de coorte. Rev. esc. enferm. USP, 52. DOl: https://doi.org/10.1590/ S1980-220X2017042403368

Zanotto, D. F., \& Assis, F. B. (2017). Perfil dos usuários de crack na mídia brasileira: análise de um jornal e duas revistas de edição nacional. Physis, 27 (03) D0l: https://doi.org/10.1590/S0103-73312017000300020 